\title{
Dendritic-cell-based immunotherapy evokes potent anti-tumor immune responses in CD105+ human renal cancer stem cells
}

\author{
Xiao-Fei Zhang ${ }^{1,2}$ | De-sheng Weng ${ }^{1,2}$ | Ke $\operatorname{Pan}^{1,2}$ | Zi-Qi Zhou ${ }^{1,2}$ | \\ Qiu-zhong Pan $^{1,2}$ | Jing-Jing Zhao ${ }^{1,2}$ | Yan Tang ${ }^{2}$ | Shan-Shan Jiang ${ }^{1,2}$ \\ Chang-Long Chen ${ }^{1,2}$ | Yong-Qiang Li $^{2}$ | Hong-Xia Zhang ${ }^{1,2}$ | Alfred E. Chang ${ }^{3}$ \\ Max S. Wicha ${ }^{3}$ | Yi-Xin Zeng ${ }^{4}$ | Qiao $\mathrm{Li}^{3}$ | Jian-Chuan Xia ${ }^{1,2}$ (iD
}

${ }^{1}$ State Key Laboratory of Oncology in Southern China, Collaborative Innovation Center for Cancer Medicine, Sun Yat-sen University Cancer Center, Guangzhou,

People's Republic of China

2 Department of Biotherapy, Sun Yat-Sen University Cancer Center, Guangzhou,

People's Republic of China

3 University of Michigan Comprehensive Cancer Center, Ann Arbor, Michigan

${ }^{4}$ Beijing Hospital, Beijing, China

\section{Correspondence}

Jian-Chuan Xia, State Key Laboratory of Oncology in Southern China, Collaborative Innovation Center for Cancer Medicine, Sun Yat-sen University Cancer Center, Guangzhou 510060, People's Republic of China.

Email: xiajch@mail.sysu.edu.cn

Qiao Li, University of Michigan Comprehensive Cancer Center, Ann Arbor, MI.

Email: qiaoli@umich.edu

Funding information

Guangdong Science and Technology Program, Grant number: 2013B021800063;

Guangdong Province Science and Technology Plan Project, Grant number:

2012A030400059; National Natural Science Foundation of China, Grant numbers: No. 81402560, No. 81472387; Gillson

Longenbaugh Foundation
Cancer stem cells (CSCs) are responsible for tumor initiation, progression, and resistance to therapeutic agents; they are usually less sensitive to conventional cancer therapies, and could cause tumor relapse. An ideal therapeutic strategy would therefore be to selectively target and destroy CSCs, thereby preventing tumor relapse. The aim of the present study was to evaluate the effectiveness of dendritic cells (DCs) pulsed with antigen derived from CD105+ human renal cell carcinoma (RCC) CSCs against renal cancer cells in vitro and in vivo. We identified "stem-like" characteristics of CD105+ cells in two human RCC cell lines: A498 and SK-RC-39. Loading with cell lysates did not change the characteristics of the DCs. However, DCs loaded with lysates derived from CD105+ CSCs induced more functionally specific active T cells and specific antibodies against CSCs, and clearly depressed the tumor growth in mice. Our results could form the basis for a novel strategy to improve the efficacy of DCbased immunotherapy for human RCC.

\section{KEYWORDS}

cancer stem cells, CD105, cellular immunotherapy, dendritic cell, renal cell carcinoma

\section{1 | INTRODUCTION}

Human tumors arise from a small subset of primary cells known as cancer stem cells (CSCs) or tumor initiating cells, ${ }^{1,2}$ which are responsible for tumor initiation, progression, and resistance to traditional therapeutic agents. ${ }^{3,4}$ CSCs have been identified in several solid tumors including human renal cell carcinoma (RCC)..$^{5-11}$ There are several hypotheses to describe the origin of CSCs, such as the accumulation of mutations, or reprogramming of tumor cells via dedifferentiation due to hypoxia and/or epithelial-to-mesenchymal 
transition (EMT). ${ }^{12-14}$ Although conventional methods, including chemotherapy and radiotherapy are able to reduce the tumor burden, and improve the survival of some cancer patients, CSCs are usually slow cycling and therefore pose an obstacle to such therapy. ${ }^{15}$ Therapies that target the pool of differentiated tumor cells but fail to eradicate CSC compartments could end up with new tumors in different locations, and lead to tumor relapse. Thus, the development of novel therapeutic strategies focusing on the elimination of CSCs could prove instrumental in preventing tumor regression and relapse. ${ }^{16}$ There have been several recent studies on CSC-targeting strategies, including focusing on specific CSC markers or molecular signaling pathways, targeting the CSC niche, manipulating miRNA expression, and inducing apoptosis or differentiation in CSCs. ${ }^{17-23} \mathrm{We}$ have described the immunological targeting of CSCs in several animal models. $^{24,25}$

Emerging CSC-targeted therapies may help understand the immune-escape-mechanisms of cancers. Current immunotherapeutic strategies are mainly based on antigens presented to effector T-cells by dendritic cells (DCs). These antigens are mostly derived from differentiated tumor cells, but may not be expressed by $\mathrm{CSCs}^{26}$ Further, CSC antigen presentation could be defective due to the downregulation of human leukocyte antigen (HLA) surface expression. ${ }^{27}$ Therefore, in a heterogeneous tumor entity, CSCs are likely not affected by current therapeutic agents and therefore cause treatment failure and disease progression. The efficacy and success of the cellular immunotherapy, using sipuleucel-T, in the treatment of metastatic prostate cancer ${ }^{28}$ has prompted the evaluation of $D C$ vaccines to treat metastatic renal cell cancer. Phase I studies of vaccines containing DCs transfected with tumor RNA or pulsed with tumor lysate showed that these were safe and effective in treating RCC either alone 29-32 $^{\text {or in }}$ combination with cytokines. ${ }^{33,34}$ Recent studies have shown that CSCs can be used as antigen sources to elicit DC-mediated CSC-specific humoral and cellular immune responses, leading to efficient antitumor immunity. ${ }^{24,25,35-39}$ These studies support the hypothesis that CSCs can be recognized and eradicated by the immune system, and provide a basis for new immunotherapeutic approaches targeting CSCs.

CD105 is a surface transmembrane molecule and act as a coreceptor for TGF-beta. It regulates cell proliferation, differentiation, and migration and has an important role in angiogenesis. ${ }^{40}$ In 2008, Bussolati et $\mathrm{al}^{11}$ proposed CD105 as a marker for tumor-initiating stem cells for the first time. They found that CD105+ cancer cells isolated from renal cancer samples display properties of stem cells such as clonogenicity and expression of the stem cell markers Nestin, Nanog, and Oct-3/4. In addition, CD105+ meningioma cells have similar increased tumorigenicity and capacity to differentiate into adipocytes and osteocytes as reported by Hu et al. ${ }^{41}$ The similar results were also found in ovarian cancer, ${ }^{42}$ glioblastoma, ${ }^{43}$ Wilms tumor cells, ${ }^{44}$ oral cancer. ${ }^{45}$

In the present study, we attempted to target human renal CSCs by using DC-based immunotherapy. Our results demonstrated the potency of DCs loaded with CSC antigen to induce cellular (cytotoxic T-lymphocytes) and humoral (specific antibody) responses that specifically target renal CSCs and thereby significantly inhibit the tumor growth in animal models.

\section{2 | MATERIALS AND METHODS}

\section{$2.1 \mid$ Cell lines}

The human RCC cell lines A498 and SK-RC-39 were purchased from the American Type Culture Collection (Manassas, VA). All cells were maintained in RPMI 1640 medium (Invitrogen, Shanghai, China) supplemented with $10 \%$ fetal bovine serum (FBS; Gibco, NY), and $1 \%$ penicillin-streptomycin at $37^{\circ} \mathrm{C}$ in a humidified atmosphere of $5 \%$ $\mathrm{CO}_{2}$ in air.

\section{2 | Cell sorting and flow cytometry}

Cell sorting was performed using MACS positive selecting kits (Miltenyi Biotec, Bergisch Gladbach, http://www.miltenyibiotec. com), according to manufacturer's instructions.

For isolation of CD105+ and CD105-cell populations, cells were magnetically labeled with the kit-provided antibody against CD105. Cells were first washed twice and subsequently resuspended in MACS buffer. An aliquot was removed and combined with $10 \mathrm{uL}$ anti-CD105 microbeads (per $10^{7}$ total cells). The cells were then incubated for $15 \mathrm{~min}$ at $2-8^{\circ} \mathrm{C}$ and subsequently washed with kit buffer. The cells were then re-suspended in 500 uL MACS buffer and CD105+ cells were isolated by magnetic separation.

For B-cell positive selection, peripheral blood mononuclear cells (PBMCs) were magnetically labeled with kit-provided antibody against CD19. And then the cells were separated according to the manufacturers instructions.

The purity of the MACS-isolated CD105+ CSCs and B-cells was assessed by flow cytometry (Beckman Coulter, Inc., Kraemer Boulevard Brea, CA, https://www.beckmancoulter.com); purity was routinely $>90 \%$. The obtained subpopulations were maintained for less than $12 \mathrm{~h}$ at $4-8^{\circ} \mathrm{C}$ until further use.

\section{3 | Sphere formation assay}

To examine the ability of the sorted CD105+ cells and CD105subpopulations to produce tumor spheres, the cells were suspended in serum-free medium (SFM) consisting of DMEM/F-12 supplemented with $20 \mathrm{ng} / \mathrm{mL}$ EGF (Epidermal Growth Factor) (PeproTech, https:// www.peprotech.com), 20 ng/mL bFGF (basic Fibroblast Growth Factor) (PeproTech, https://www.peprotech.com), and B27 (1×) (Invitrogen, http://www.thermofisher.com). In all experiments, cells were incubated at $37^{\circ} \mathrm{C}$ in a humidified $5 \% \mathrm{CO}_{2}$ atmosphere for a maximum of 12 days.

\section{4 | Tumor genesis assay}

The limiting dilution assay was carried out to compare the tumor induction potency of the renal carcinoma cell subpopulations. Briefly, 4-week-old female BALB/c nude female mice (Medical Experimental Animal Center of Guangdong Province, Guangzhou, China, http://www. gdmlac.com.cn) were challenged with four dilutions of both sorted tumor cell subpopulations (CD105+ and CD105- RCC cells from A498 
and SK-RC-39). The number of injected cells was 10000,100000 , 1000000 , or 5000000 for each mouse in the groups. Sorted cells were suspended in $100 \mu \mathrm{L}$ PBS containing 50\% Matrigel (BD Biosciences, Lake Franklin, NJ, http://www.bd.com), chilled on ice, and then injected subcutaneously into the flanks of the mice via a 26-gauge needle using a 1-mL syringe. Typically, CD105+ and CD105- cells were injected into the left and right sides of the same animal. The tumorigenic potential of each subpopulation was followed daily for 12 weeks. Tumor size in cubic millimeters was assessed weekly with calipers and was calculated as tumor size $=\left(\right.$ largest diameter $\times$ smallest diameter $\left.{ }^{2}\right) / 2$.

\subsection{Immunofluorescence}

Indirect immunofluorescence was performed on cells cultured on chamber slides. Sorted CD105+ and CD105- cells were fixed in 3.5\% paraformaldehyde and permeabilized with Hepes-Triton X-100 buffer. The following antibodies were used: anti-SOX2, anti-Nanog, anti-c-Myc, and anti- KLF4 (Cell Signaling Technology, Trask Lane Danvers, MA, https://www.cellsignal.com). Alexa Fluor 594 anti-rabbit IgG (Molecular Probes, https://www.thermofisher.com) were used as secondary antibody. DAPI dye (Sigma, Darmstadt, Germany, http://www.sigmaaldrich. com) was added for nuclear staining.

\section{6 | Analysis of cell cycle status of CSCs}

For cell cycle analysis, the sorted CD105+ and CD105- RCC cells were collected and washed with ice-cold PBS for 5 min by centrifugation at $125 \times$ g. The cells were then fixed with $75 \%$ ethanol at $-20^{\circ} \mathrm{C}$ overnight. The cells were then treated with RNase at $37^{\circ} \mathrm{C}$ for 30 min and stained with propidium iodide for $60 \mathrm{~min}$ at $4^{\circ} \mathrm{C}$ in the dark. Finally, cells were analyzed with a Flow Cytometer (Beckman Coulter, https://www. beckmancoulter.com) according to manufacturer's instructions.

\section{7 | Cell viability assay}

Cell viability was determined using a sensitive colorimetric assay (MTS; Promega, https://cn.promega.com). Cells $\left(8 \times 10^{3}\right.$ cells per well) were seeded in flat-bottom 96-well plates. After incubation for $12 \mathrm{~h}$, cells were treated with varying concentrations of cisplatin. After further incubation for $48 \mathrm{~h}, 20 \mu \mathrm{L}$ MTS solution was added to each well, followed by $4 \mathrm{~h}$ incubation at $37.5^{\circ} \mathrm{C}$ Absorbance at $490 \mathrm{~nm}$ was measured using a SpectraMax M5 plate reader (Molecular Devices, https://www.moleculardevices.com). Relative cell viability was calculated as percentage of untreated controls. Drug sensitivity was determined from three separate experiments and expressed as the drug concentration required to inhibit proliferation by $50 \%$ (IC50), by using standard curve-fitting routines (GraphPad Software, Inc, http://www.graphpad.com).

\section{8 | Apoptosis assay}

For the cell apoptosis assay, the CD105+, and CD105- cells that were treated with $10 \mu \mathrm{mol} / \mathrm{L}$ cisplatin for $12 \mathrm{~h}$ were harvested, washed twice with ice-cold PBS, and stained with Annexin V-FITC, and PI (Bestbio, http://bestbio.bioon.com.cn) according to manufacturer's instructions. Cells were analyzed using a Flow Cytometer (Beckman Coulter, https:// www.beckmancoulter.com) according to manufacturer's instructions.

\subsection{Generation of monocyte-derived dendritic cells, $T$ cells, and $B$ cells}

PBMCs were separated from $50 \mathrm{~mL}$ peripheral blood obtained from HLA-A* 02 healthy donors by Ficoll density gradient centrifugation (GE Health, http://www.gehealthcare.com), and were incubated in six-well culture plates at $37^{\circ} \mathrm{C}$ for $1 \mathrm{~h}$ in RPMI 1640 with $5 \%$ human $A B$ serum. After incubation for about $2 \mathrm{~h}$, the adherent PBMCs were cultured in Quantum 007 Lymphocytes medium (PAA, Freiburg, Germany, http:// www.paa.com) containing $1000 \mathrm{U} / \mathrm{mL}$ granulocyte macrophage colony stimulating factor (GM-CSF) and $500 \mathrm{U} / \mathrm{mL}$ interleukin-4 (IL-4) (both from Life Technologies, Guangzhou, People's Republic of China). On day 5, DCs were harvested by dispenser and enriched by Opti-Prep density gradient medium. Lysates of unsorted renal cancer cells, CD105+ CSCs, or CD105- non-CSCs were added to DCs at a 1:3 cell equivalent ratio. On day 6 , to mature the DCs and help them upregulate the expressions of costimulatory molecules, OK-432 (Shandong Lukang Pharmaceutical Co. Ltd. http://www.lkpc.com) was added into the culture medium at $0.1 \mathrm{KE} / \mathrm{mL}$.

The non-adherent cells were divided into two aliquots. One was cultured in RPMI 1640 with 20 U/mL IL-2 (Peprotech, Suzhou, People's Republic of China, http://www.peprotechchina.com) and 10\% human $A B$ serum, to obtain autologous $T$ cells. The other was magnetically labeled with kit-provided antibody against CD19. CD19 B cells were positively selected as described in the "Cell sorting" section. Purified B cells were cultured in complete medium supplemented with $5 \mu \mathrm{g} / \mathrm{mL}$ LPS(Beyotime, http://www.bio-equip.cn), $2 \mu \mathrm{g} / \mathrm{mL}$ anti-CD40 (Sino Biological Inc, http://sbi2007.en.ec21.com), and $60 \mathrm{IU} / \mathrm{mL}$ IL-2.

Half of the total culture medium was changed every two days. Non-adherent and loosely adherent cells were harvested after 6 days.

\subsection{0 | Preparation of cell lysates loaded-DCs}

To prepare tumor cell lysates, unsorted renal cancer cells, sorted CD105+ CSCs, or CD105- cells were suspended at a concentration of $1 \times 10^{7} / \mathrm{mL}$ in PBS. Cells were lysed by five rapid freeze-thaw cycles in a $37^{\circ} \mathrm{C}$ water bath and liquid nitrogen. After centrifugation (12000 rpm for $20 \mathrm{~min}$ ), tumor cell lysates were collected. Lysates of A498 unsorted renal cancer cells, A498 CD105+ CSCs, or A498 CD105- non-CSCs were added to DCs at a 1:3 cell equivalent ratio. And lysates of SK-RC-39 unsorted renal cancer cells, SKRC-39 CD105+ CSCs, or SK-RC-39 CD105- non-CSCs were also added to DCs at a $1: 3$ cell equivalent ratio. The DCs were then incubated at $37^{\circ} \mathrm{C}$ for $24 \mathrm{~h}$ with $5 \% \mathrm{CO}_{2}$. After incubation, the $\mathrm{A} 498$ unsorted renal cancer cell lysate-pulsed DCs (A498 Unsorted_DCs), A498 CD105+ lysate-pulsed DCs (A498 CD105 + _DCs), or A498 CD105- lysate-pulsed DCs (A498 CD105_DC) and the SK-RC-39 unsorted renal cancer cell lysate-pulsed DCs (SKRC-39 Unsorted_DCs), SK-RC-39 CD105+ lysate-pulsed DCs (SK-RC-39 CD105+_DCs), or SK-RC-39 CD105- lysate-pulsed DCs (SK-RC-39 CD105-_DC) were used in the subsequent experiments. 


\subsection{Phenotypic analysis of cell lysates loaded-DCs}

Cell lysates loaded-DCs were harvested, washed, and re-suspended in FACS buffer (PBS containing 2\% FCS). The cells were then stained with anti-CD80, anti-CD83, anti-CD86, anti-HLA-DR, and anti-HLA-ABC antibodies (all from Biolegend, http://www.biolegend.com) for $20 \mathrm{~min}$ at $4^{\circ} \mathrm{C}$ and analyzed for the expression of the above-mentioned phenotypic markers, by flow cytometry.

\subsection{2 | Functional analysis of cell lysates loaded-DCs (enzyme-linked immunosorbent assay)}

Cell lysates loaded-DCs $\left(2 \times 10^{5}\right)$ were cultured in a 48-well plate in $1 \mathrm{~mL} \mathrm{DC}$ medium. Supernatants were harvested after $24 \mathrm{~h}$, and IL12 p70 production was determined using a commercially available ELISA kit (Neobioscience Technology, http://www.made-in-china. com) according to the manufacturer's protocol.

\subsection{3 | Cytotoxic T Lymphocyte (CTL) generation}

T cells from the same healthy donor were used as responder cells and stimulated with cell lysates loaded-DCs at an S:R ratio of 1:10. Cells were cultured in Quanta-007 lymphocyte culture medium containing IL-2 $(20 \mathrm{U} / \mathrm{mL})$ and IL-7 $(5 \mathrm{ng} / \mathrm{mL}$; Peprotech). Half the medium was replaced every other day with fresh medium. On day 7, CTLs were harvested and used for phenotypic and functional studies.

\subsection{Cytotoxicity assay}

After 7 days of co-culture, CTLs were used for cytotoxicity assays. T cells were divided into four groups; (i) T cells stimulated with CD105+ cell lysateloaded DCs (A498 CD105 + _CTLs, SK-RC-39 CD105+_CTLs); (ii) T cells stimulated with CD105- cell lysate-loaded DCs (A498 CD105-_CTLs, SKRC-39 CD105- _CTLs); (iii) T cells stimulated with unsorted cell lysateloaded DCs (A498 Unsorted_CTLs, SK-RC-39 Unsorted_CTLs); and (iv) T cells stimulated with control mature DCs alone (Con_CTLs). The cytotoxicity assays were conducted using the Cyto Tox 96 Non-Radioactive Cytotoxicity Assay Kit according to the protocol provided (Promega). For A498 CD105 +_CTLs, A498 CD105- _CTLs, A498 Unsorted_CTLs, and Con_CTLs, the targets were A498 CD105+ CSCs, A498 CD105- non-CSCs, and A498 unsorted cells, respectively. For SK-RC-39 CD105+_CTLs, SK-RC-39 CD105-_CTLs, SK-RC-39 Unsorted_CTLs, and Con_CTLs, the targets were SK-RC-39 CD105+ CSCs, SK-RC-39 CD105- non-CSCs, and SK-RC-39 unsorted cells, respectively. After washing, effector T cells were added to the targets at E:T ratios of 3:1, 10:1, or 30:1.

\subsection{5 | Winn assay}

Unsorted RCC cells $\left(2 \times 10^{6}\right)$ were mixed with $2 \times 10^{7}$ CTLs and injected subcutaneously into BALB/c nude mice. A control group of mice was injected with unsorted renal cells alone. Tumor size was assessed every 2 days with calipers. All mice were sacrificed at 12 weeks, and xenografts were removed for IHC and FACS analyses.

\subsection{6 | Intracellular cytokine analysis}

For intracellular staining, the CTLs (alone or cocultured with CD105+ CSCs) were stimulated for $4 \mathrm{~h}$ in the presence of $50 \mathrm{ng} / \mathrm{mL}$ phorbol myristate acetate, $500 \mathrm{ng} / \mathrm{mL}$ ionomycin (Sigma), and $2 \mu \mathrm{M}$ monensin (GolgiStop, BD Biosciences). The cells were incubated with anti-CD4 and anti-CD8 for surface staining, followed by intracellular staining using anti-TNF- $\alpha$, anti-IFN- $\gamma$, anti-Perforin, or anti-Granzyme B (all from Biolegend) in FIX/PERM buffers (BD Pharmingen, http://www. yubiotech.com) according to the manufacturers' instructions. The samples were evaluated on an FC500 flow cytometer (Beckman Coulter, https://www.beckmancoulter.com) and analyzed using CXP Software (Beckman Coulter, https://www.beckmancoulter.com).

\subsection{7 | B cell activation}

B cells from the same healthy donor were used as responder cells and stimulated with cell lysates loaded-DCs at an S:R ratio of 1:10. Cells were cultured in complete medium supplemented with $5 \mu \mathrm{g} / \mathrm{mL}$ LPS, $2 \mu \mathrm{g} / \mathrm{mL}$ anti-CD 40 , and $60 \mathrm{IU} / \mathrm{mL}$ IL-2. Half the medium was replaced every other day with fresh medium. On day 7 , the culture supernatants were collected and used for functional studies.

\subsection{CSC binding by immune supernatant}

The concentrations of IgG in the mixed B cell culture supernatants were detected using ELISA (Westang, http://westang.bioon.com.cn) before we performed the binding assays. Based on the ELISA results (concentration of $\operatorname{lgG}$ ), we determined the volume required from each mixed sample to ensure that an equal quantity of IgG from each coculture activated group was present to assay binding to CD105+ CSCs, Unsorted cells, or CD105- non-CSCs. Unsorted RCC cells, sorted CD105+ CSCs, or CD105- non-CSCs were incubated with the appropriate volume of immune supernatants collected from the cocultured B cells comprising an equal quantity of IgG. They were then incubated with FITC-conjugated anti-human IgG. The binding of supernatant antibody to CD105+ CSCs, CD105- non-CSCs, or Unsorted cells was subsequently assessed using flow cytometry.

\subsection{9 | Antibody- and complement-mediated cytotoxicity against CSCs}

Viable CD105+ CSCs, CD105- non-CSCs, or Unsorted cells were incubated with immune supernatants obtained from activated $B$ cells cocultured with cell lysates loaded-DCs. The cells were then incubated with rabbit complement (Merck) for another $1 \mathrm{~h}$. Trypan blue staining was used to assess cell lysis, which was expressed as: percent viable cells $=$ number of viable cells after immune supernatant and complement incubation $/ 10^{5}$.

\subsection{Statistical analysis}

All statistical analyses were performed using SPSS version 19.0 (SPSS Inc., Chicago, IL). The Student $t$-test or Wilcoxon test was used to 
analyze the differences between quantitative variables. The Pearson $\chi^{2}$ test or Fisher exact test was used to analyze the differences between qualitative variables. All tests were two-sided, and $P<0.05$ was considered statistically significant.

\section{3 | RESULTS}

\subsection{The CD105+ fraction of human renal cell carcinoma cells was considered as cancer stem cells}

The RCC subpopulations were analyzed and sorted according to the expression of CD105. Prior to MACS, the frequency of CD105+ cells was $8.3 \pm 1.3 \%$ for $A 498$ ( $n=3$ repeat experiments) and $4.9 \pm 1.7 \%$ ( $n=3$ repeat experiments) for SK-RC-39. Then cells were sorted by MACS and the purity of the two fractions was more than $92 \%$ (Supplementary Fig. S1). The sorted CD105+ and CD105- cells from the A498 and SK-RC-39 cell lines were studied for spheroid formation potency in SFM. When plated in sphere-generating medium, the CD105+ cells were able to grow in non-adhesive condition and generate spheres; however, most of the CD105- cells did not survive in the SFM (Fig. 1A). Moreover, we determined the tumorigenic ability of CD105+ cells; serial dilutions of sorted CD105+ and CD105- cells from the A498 and SK-RC-39 cell lines were injected subcutaneously into nude mice. The results showed that the tumorigenicity of the CD105+ (red arrows) renal cancer cells was much higher than that of the CD105- (black arrows) cells (Fig. 1B). Because of the enhanced tumorigenic and spheroid formation abilities of CD105+ cells, they were considered as renal CSCs.

In addition, we found that CD105+ renal cancer cells exhibit stemcell-like properties such as the significant expression of the established stem cell markers Sox2, Nanog, c-Myc, and KLF4 (Fig. 1C).

The quiescent state is likely necessary for the self-renewal of stem cells including CSCs. ${ }^{46}$ We therefore analyzed the cell cycle status of CD105+ and CD105- cells. The proportion of cells arrested at the G0/ G1 phase in the CD105+ population was significantly higher than the corresponding percentages in the CD105- population (Fig. 1D).

Another main feature of CSCs is a strong resistance to chemotherapeutic drugs ${ }^{43,47}$; the degree of resistance to cisplatin could be used to differentiate CSC-like mammary tumor cells from normal mammary cells. ${ }^{48}$ Here, we treated CD105+ and CD105- cells with various doses of cisplatin and performed a MTT assay to assess their growth capacity after $12 \mathrm{~h}$ of incubation (Fig. 1E). Both cell populations showed a significant resistance to cisplatin; however, CD105- cells showed significantly lower cisplatin resistance. What's more, treatment with cisplatin at $10 \mu \mathrm{mol} / \mathrm{mL}$ induced significantly more apoptosis in CD105- cells than in CD105+ cells.

\subsection{Generation and analysis of cell lysates loaded-DCs}

Because CD105+ renal cancer cells presented characteristics of tumor stem cells in human renal cancer, we hypothesized that these cells could be an immunological target for RCC CSC-directed immunotherapy.
Human peripheral blood mononuclear cell-derived DCs were loaded with CD105+ cell lysates, CD105- cell lysates, unsorted renal cancer cell lysates, or not loaded with anything (negative control). As shown in Supplementary Fig. S2A (A498 cell line) and Supplementary S2B (SKRC-39 cell line), mature DCs loaded with CD105+ cell lysates (CD105 +_DCs), CD105- cell lysates (CD105-_DCs,) or unsorted renal cancer cell lysates (Unsorted_DCs) expressed high levels of the costimulatory and activation markers CD80, CD83, CD86, HLA-ABC, and HLA-DR, which were similar to those observed in the mature DCs without cell lysate (Con_DCs). To determine whether the lysate-loaded DCs were functionally active, IL-12p70, which is considered important for the Th1 response, was detected in the culture supernatants of monocytederived DCs after loading with cell lysates. The production of IL-12p70 was not significantly altered in DCs loaded with CD105+ lysates compared to unloaded mature DCs or DCs loaded with CD105- cell lysate and unsorted renal cancer cell lysates (Supplementary Fig. S2C,D).

\subsection{Cell lysates loaded-DCs potently stimulate autologous $\mathrm{T}$ cells}

To determine whether cell lysate-loaded DCs could activate T cells, we determined the number of effector $T$ cells after incubating autologous $T$ cells with the different treated DCs, using IFN- $\gamma$ expression as a marker of functionally active T cells. A498 CD105+_DCs, A498 CD105- _DCs, and A498 Unsorted_DCs stimulated the production of significantly higher numbers of IFN- $\gamma$-expressing T cells than did Con_DCs (Fig. 2A, C). SK-RC-39 CD105+_DCs, SK-RC-39 CD105-_DCs, and SK-RC-39 Unsorted_DCs stimulated the production of significantly higher numbers of IFN- $\gamma$-expressing T cells than did Con_DCs (Fig. 2B,D). However, the percentage of IFN- $\gamma$ production level by CD8+ CTL cells were not significantly different among $T$ lymphocytes stimulated by CD105+_DCs, CD105- _DCs, or Unsorted_DCs both in A498 cell line and SK-RC-39 cell lines. Thus, cancer cell lysate-loaded DCs are capable of inducing greater numbers of functionally active $T$ cells.

\section{4 | Preferential destruction of the CD105+ RCC CSC population by $T$ cells stimulated with CD105 +_DCs in vitro and in vivo}

Next, we tested the CTL activity of T cells stimulated with CD105 +_DCs (CD105+_CTLs), CD105- _DCs (CD105- _CTLs), Unsorted_DCs (Unsorted_CTLs), or Con-DCs (Con_CTLs) against CD105+ CSCs, Unsorted cancer cells, and CD105- non-CSCs, respectively by using the LDH release assay. CTLs induced by A498 CD105+_DCs exhibited the highest levels of cytotoxicity against A498 CD105+ CSCs, lower activity against the bulk population of A498 Unsorted cells and sorted A498 CD105- non-CSCs. CTLs induced by SK-RC-39 CD105+_DCs exhibited the highest levels of cytotoxicity against SKRC-39 CD105+ CSCs, lower activity against the bulk population of SKRC-39 Unsorted cells and sorted SK-RC-39 CD105- non-CSCs (Fig. 3A). Lysis by CTLs induced with Con_DCs was significantly less effective, regardless of whether the target was CD105+ cells, CD105cells, or bulk unsorted cells both in A498 cell line and SK-RC-39 cell 
A

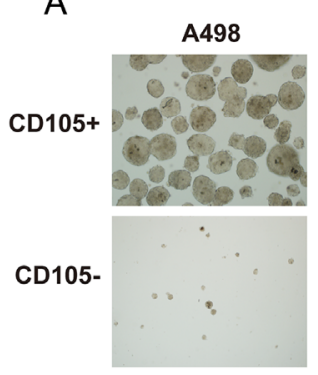

C
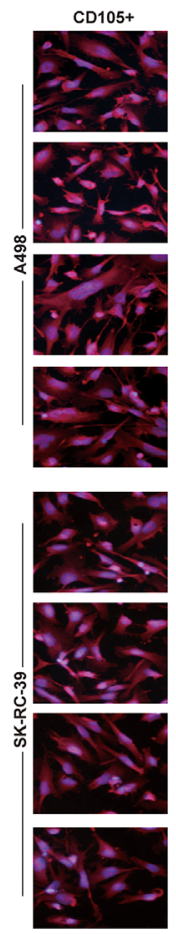

SK-RC-39

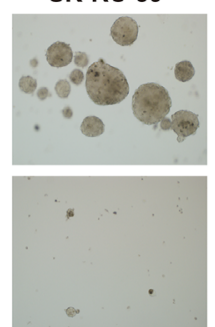

CD105-
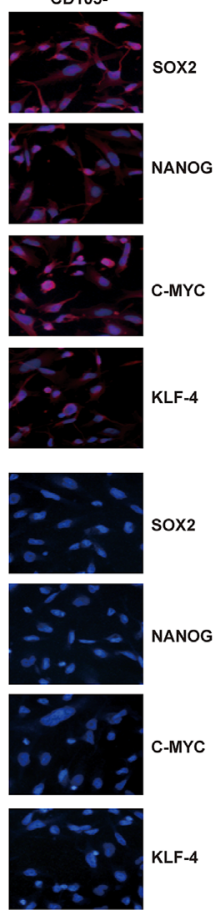

B

\begin{tabular}{ccccc}
\multicolumn{5}{c}{ A498 } \\
\hline \multirow{2}{*}{ Type } & \multicolumn{4}{c}{ Number } \\
\cline { 2 - 5 } & $\mathbf{1 \times 1 0 ^ { 4 }}$ & $\mathbf{1 \times 1 0 ^ { 5 }}$ & $1 \times 10^{6}$ & $\mathbf{5 \times 1 0 ^ { 6 }}$ \\
\hline CD105+ & $0 / 3$ & $2 / 3$ & $3 / 3$ & $5 / 5$ \\
\hline CD105- & 0 & 0 & $1 / 3$ & $5 / 5$ \\
\hline
\end{tabular}

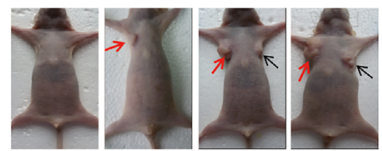

\begin{tabular}{|c|c|c|c|c|}
\hline \multicolumn{5}{|c|}{ SK-RC-39 } \\
\hline \multirow{2}{*}{ Type } & \multicolumn{4}{|c|}{ Number } \\
\hline & $1 \times 10^{4}$ & $1 \times 10^{5}$ & $1 \times 10^{6}$ & $5 \times 10^{6}$ \\
\hline CD105+ & $1 / 5$ & $3 / 5$ & $5 / 5$ & $5 / 5$ \\
\hline CD105- & 0 & 0 & $2 / 5$ & $5 / 5$ \\
\hline
\end{tabular}

D
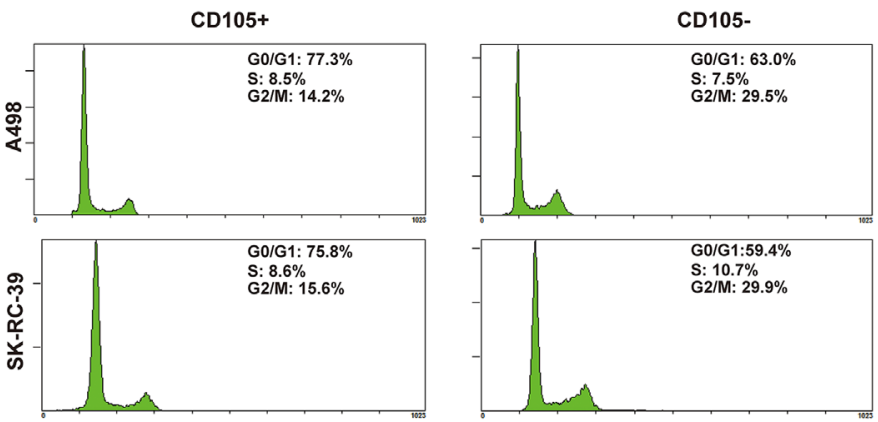

$\mathrm{E}$
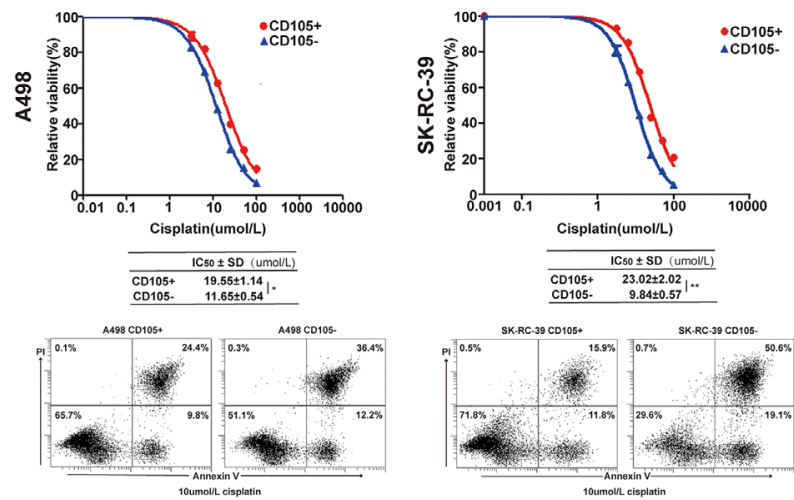

FIGURE 1 Characterization of CD105+ cancer stem cells obtained from human renal cell carcinoma cells. (A) Micrograph representative of spheres generated by culture of CD105+ vs CD105- clones (A498 and SK-RC-39); original view $\times 200$. (B) Tumorigenicity of CD105+ cells determined by xenograft assay. The indicated numbers of cells were subcutaneously injected into nude mice and tumor formation was monitored weekly. A498 CD105+ (or SK-RC-39 CD105+) cells and A498 CD105- (or SK-RC-39 CD105-) cells were subcutaneously inoculated into the left and right flanks of mice, respectively. (C) Representative immunofluorescence expression of the stem cell markers SOX2, NANOG, C-MYC, and KLF-4 by CD105+ vs CD105- cell clones; original view $\times 650$. Nuclei were counterstained with DAPI. (D) Cytofluorimetric analysis of cell cycle status (G0/G1, S, G2/M) of CD105+ vs CD105- cells. (E) CD105+ cells showed enhanced chemoresistance. Up: A498 and SK-RC-39 CD105+ cells were more sensitive to cisplatin. Middle: Sensitivity of CD105+ vs CD105- cells to cisplatin. IC50, median inhibitory concentration; SD, standard deviation. Down: $10 \mu \mathrm{mol} / \mathrm{m}$ cisplatin induced less apoptosis in CD105+ cells than in CD105- cells. $\left({ }^{*} P<0.05,{ }^{* *} P<0.01\right)$

lines (Fig. 3A). Thus, we concluded that A498 CD105+ CSCs could be preferential destruction by A498 CD105+_CTLs, but not A498 CD105- _CTLs, or A498 Unsorted_CTLs; SK-RC-39 CD105+ CSCs could be preferential destruction by SK-RC-39 CD105+_CTLs, but not SK-RC-39 CD105- _CTLs, or SK-RC-39 Unsorted_CTLs.

To confirm the anti-tumor effects of T cells induced by CD105+ cell lysate-loaded DCs, unsorted renal cancer cells mixed with different CTLs were injected subcutaneously into nude mice. The same number of renal cancer cells suspended in PBS was injected subcutaneously into nude mice as controls. At week 12 after injection, the mice were sacrificed and the tumors were extracted for flow cytometry and IHC analysis. The results showed that CTLs induced by A498 CD105+_ DCs significantly delayed tumor growth in A498 renal cancer xenografts models (Fig. 3B[a, b]). And CTLs induced by SK-RC-39 CD105+_ DCs significantly delayed tumor growth in SK-RC-39 renal cancer xenografts models (Fig. 3C[a, b]). This inhibition was consistent with a significant reduction of residual CD105+ cell content compared with tumors from the control group (Fig. 3B[c],C[c]).

\section{5 | CD105+_CTLs could recognize HLA-A2+ CD105 peptide and lyse CD105+ CSC populations}

To determine the mechanism underlying CD105+_CTL-mediated reduction of $\mathrm{CD} 105+\mathrm{CSC}$, we determined whether CD105+_CTLs could release specific anti-tumor cytokines against CD105+ CSCs. We 
A
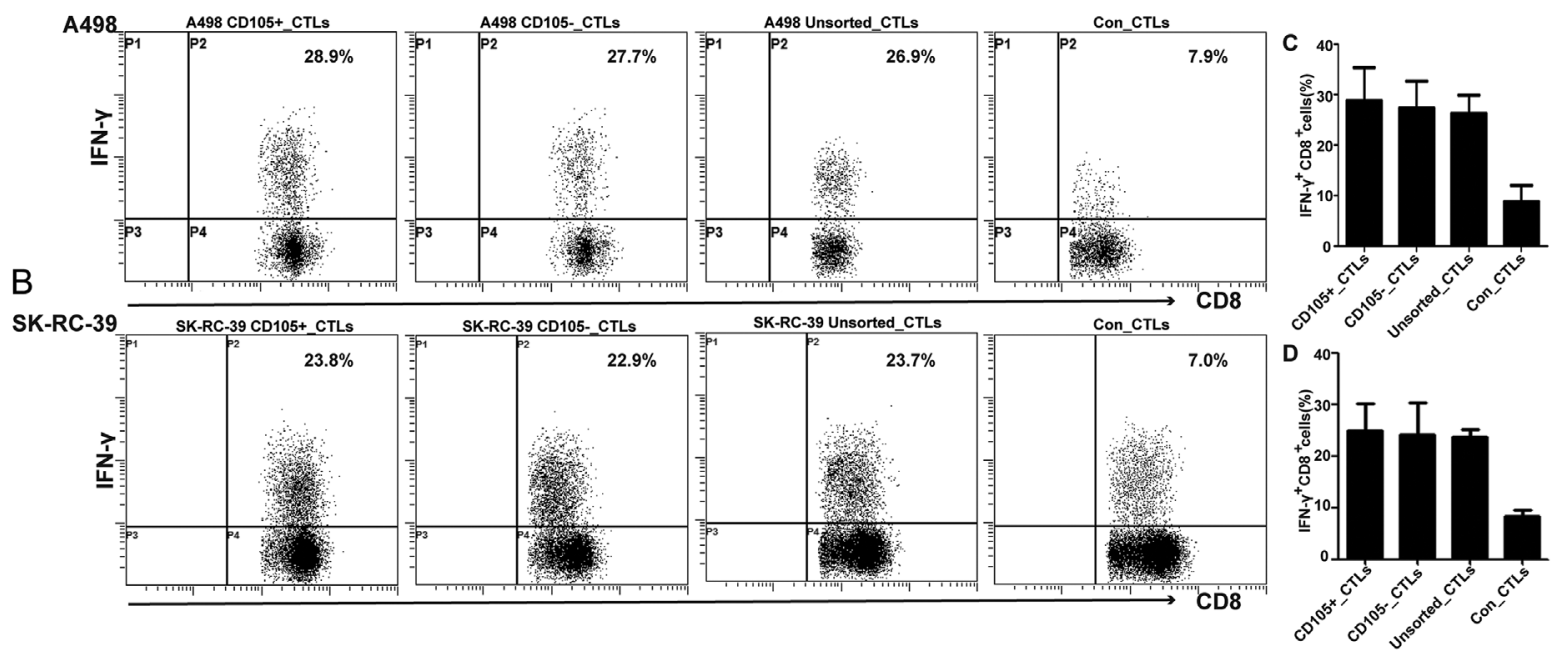

FIGURE 2 Characterization of T cells stimulated by untreated or cancer cell lysates loaded DCs. (A,B) Expression of IFN- $\gamma$ in stimulated CD8 T cells was measured by flow cytometry. (A) Autologous T cells stimulated for 7 days with untreated mature DCs (Con-CTLs), A498 CD105+ CSCs lysate-loaded DCs (A498 CD10+_CTLs), A498 Unsorted renal cancer cell lysate-loaded DCs (A498 Unsorted_CTLs), and A498 CD105- non-CSC lysate-loaded DCs (A498 CD105- _CTLs) were double stained with monoclonal antibodies against IFN- $\gamma$ and CD8, and analyzed by flow cytometry. (B) Autologous T cells stimulated for 7 days with untreated mature DCs (Con-CTLs), SK-RC-39 CD105+ CSCs lysate-loaded DCs (SK-RC-39 CD105+_CTLs), SK-RC-39 Unsorted renal cancer cell lysate-loaded DCs (SK-RC-39 Unsorted_CTLs), and SKRC-39 CD105- non-CSC lysate-loaded DCs (SK-RC-39 CD105-_CTLs) were double stained with monoclonal antibodies against IFN- $\gamma$ and $\mathrm{CD} 8$, and analyzed by flow cytometry. (C,D), The percentages of IFN- $\gamma$-positive CD8 cells from three experiments are shown in the bar graph; C for A 498 cell line, D for SK-RC-39 cell line. Data are presented as mean \pm SD. CTLs, cytotoxic T lymphocytes; DCs, dendritic cells; IFN- $\gamma$, interferon gamma

found that, after co-culture of A498 CD105+ CSCs with A498 CD105 +_CTLs, A498 CD105- _CTLs, A498 Unsorted_CTLs, or Con_CTLs, A498 CD105+_CTLs secreted significantly high levels of TNF-a, Perforin, Granzyme B than A498 CD105- _CTLs, A498 Unsorted_CTLs, and Con_CTLs did (Fig. 4A-D). After co-culture of SK-RC-39 CD105+ CSCs with SK-RC-39 CD105+_CTLs, SK-RC-39 CD105_CTLs, SK-RC-39 Unsorted_CTLs, or Con_CTLs, SK-RC-39 CD105 + +CTLs secreted significantly high levels of TNF-a, Perforin, Granzyme B than SK-RC-39 CD105- _CTLs, SK-RC-39 Unsorted_CTLs, and Con_CTLs did (Fig. 4E-H). The comparisons of differences of cyctokines released by CTLs after co-culture with CD105+ CSCs were shown in Fig. 4A-D (A498) and E-H (SK-RC-39). Therefore, stimulation with A498 CD105+ cell lysate-loaded DCs could induce specific CTL activity against A498 CD105+ RCC CSCs and stimulation with SK-RC-39 CD105+ cell lysate-loaded DCs could induce specific CTL activity against SK-RC-39 CD105+ RCC CSCs.

To determine whether the CTLs stimulated with CD105+_DCs could recognize CD105+ CSCs specifically, we performed tetramer analyses by using CD105 antigen tetramers. As shown in Fig. 4l(i), CD105+ antigen-specific CTLs were present in the A498 CD105 +_DCs stimulated T cells. As shown in Fig. 4J(j), CD105+ antigenspecific CTLs were present in the SK-RC-39 CD105 + _DCs stimulated $T$ cells. The percentages of antigen-specific CTLs were $~ 30 \%$. Clearly, optimization of peptide and tetramer designs and conditions will likely improve the detection of antigen-specific CTL generation.

Together, these results provide direct experimental evidence that RCC CD105+ CSCs can be eliminated by CTLs in vitro and in vivo.
Targeting CSCs/CICs could provide a novel therapeutic paradigm with important clinical implications.

\section{6 | CD105+_DCs modulates humoral responses specifically targeting CD105+ RCC CSCs}

To determine whether the CD105+_DCs could reinforce humoral responses specifically targeting CD105+ CSCs, we evaluated CD105 + _DCs-induced antibody immune responses against CD105+ CSCs. After co-culture of autologous B cells with the different treated DC populations, ELISA analysis was performed to detect IgG antibodies in the $B$ cell culture supernatant. To test the specificity of the CD105 +_DCs-primed antibody, we assessed the binding of the immune supernatant to CD105+ CSCs, Unsorted renal cancer cells, and CD105 - non-CSCs. As shown in Fig. 5A, culture supernatant of B cells cocultured with various $A 498$ renal cancer cell lysate loaded DCs were used to test their binding to A498 CD105+ CSCs (left column), A498 Unsorted renal cancer cells (middle column), and A498 CD105 nonCSCs (right column). As shown in Fig. 5B, culture supernatant of B cells cocultured with various SK-RC-39 renal cancer cell lysate loaded DCs were used to test their binding to SK-RC-39 CD105+ CSCs (left column), SK-RC-39 Unsorted renal cancer cells (middle column), and SK-RC-39 CD105 non-CSCs (right column). Fluorescein isothiocyanate (FITC)-labeled anti-human IgG secondary antibody was then used for detection of immunoreactivity via fluorescence cytometry. As shown in Fig. 5C, A498 CD105+_DCs-primed immune supernatant bind to A498 CD105+ CSCs significantly more than supernatant 

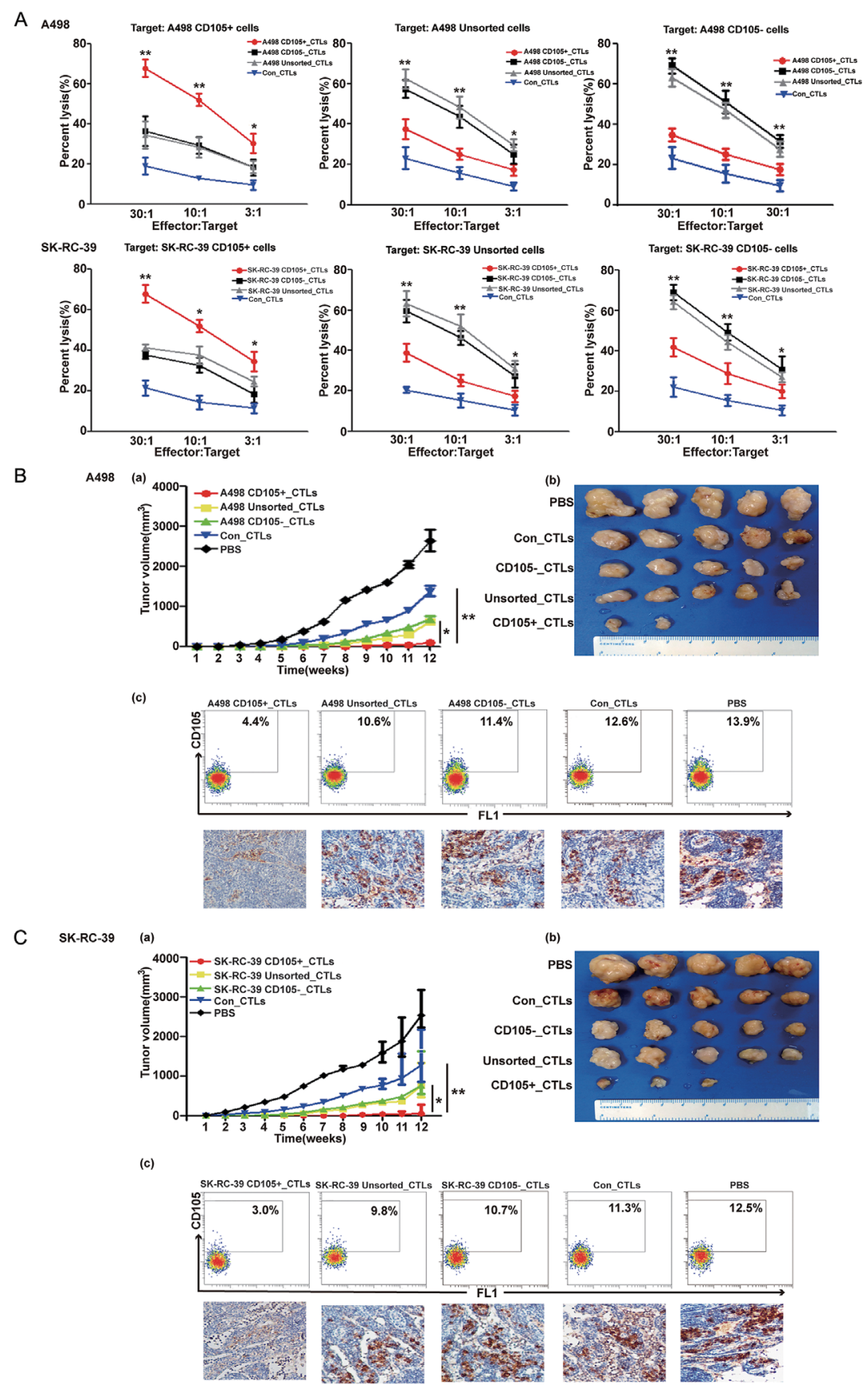

FIGURE 3 Preferential killing of RCC CSCs by CTL cells induced with CD105+ cell lysate-loaded DCs in vitro and in vivo. (A) up: T cells stimulated for 7 days with A498 CD105+_ DCs (A498 CD105+_CTLs), A498 Unsorted_DCs (A498 Unsorted_CTLs), A498 CD105- _ DCs (A498 CD105- _CTLs), or control mature DCs (Con-CTLs) were incubated with A498 CD105+, A498 CD105-, or A498 Unsorted renal cacner cells; (A) bottom: T cells stimulated for 7 days with SK-RC-39 CD105+_ DCs (SK-RC-39 CD105+_CTLs), SK-RC-39 Unsorted_DCs (SK-RC-39 Unsorted_CTLs), SK-RC-39 CD105- _ DCs (SK-RC-39 CD105- _CTLs), or control mature DCs (Con-CTLs) were incubated with SK-RC-39 CD105+, SK-RC-39 CD105-, or SK-RC-39 Unsorted SK-RC-39 renal cancer cells at ratios of 3:1, 10:1, and 30:1. CTL activity was measured in triplicate using Cyto Tox 96 Non-Radioactive Cytotoxicity Assay Kit. The results are presented as the mean \pm SD. ${ }^{*} P<0.05$; ${ }^{* *} P<0.01$. (B) A498 renal cancer cells mixed with A498 CD105+_CTLs, A498 Unsorted_CTLs, A498 CD105-_CTLs, Con_CTLs, or PBS were injected subcutaneously into nude mice. (C) SK-RC-39 renal cancer cells mixed with SK-RC-39 CD105+_CTLs, SK-RC-39 Unsorted_CTLs, SK-RC-39 CD105-_CTLs, Con_CTLs, or PBS were injected subcutaneously into nude mice. (a) Tumor sizes were determined in individual mice by measurements of two opposing diameters and are presented as tumor areas in $\mathrm{mm}^{2}$. Points, mean for each group of mice; bars, SD. Statistical significance of tumor sizes was calculated using two-way ANOVA $\left({ }^{*} P<0.05\right.$; $\left.{ }^{* *} P<0.01\right)$. (b) After 12 weeks, mice were sacrificed and the tumors were extracted. (c) The tumors of each group were immediately mechanically disaggregated and filtered to prepare signal cell suspensions or made into paraffin sections and used for immunohistochemical analysis. The CD105+ cells in the tumors were analyzed by flow cytometry and IHC. CTLs, cytotoxic T lymphocytes; DCs, dendritic cells; PBS; phosphate buffered saline 
A498

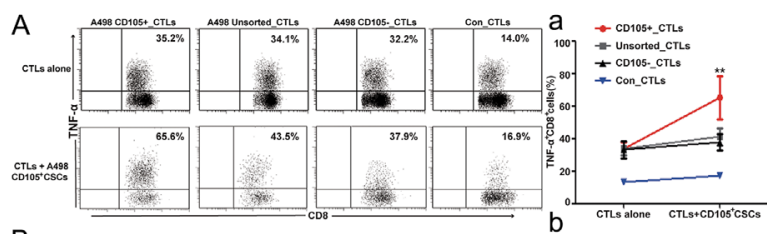

B

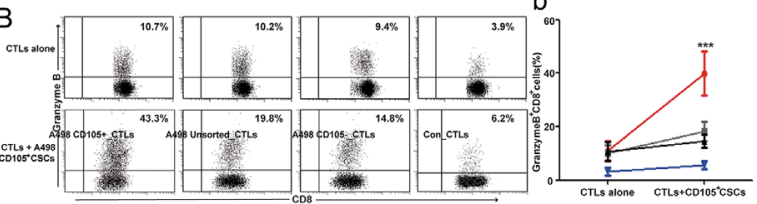

C

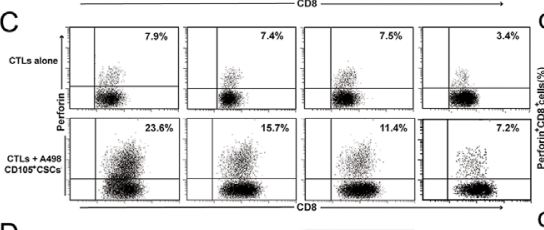

D
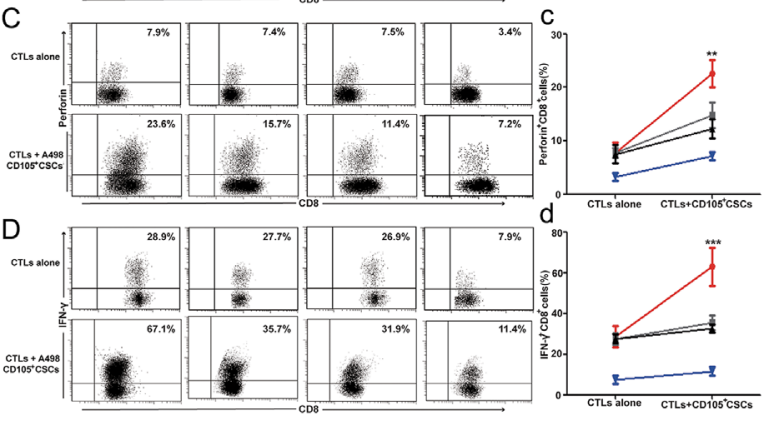
$d_{807}^{0}$
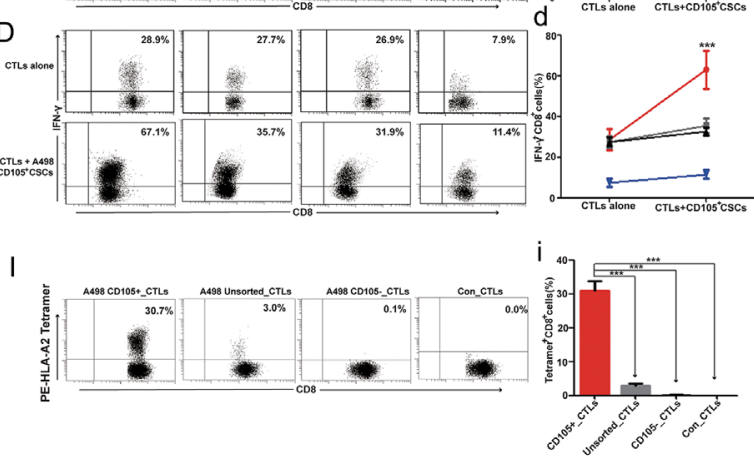

SK-RC-39
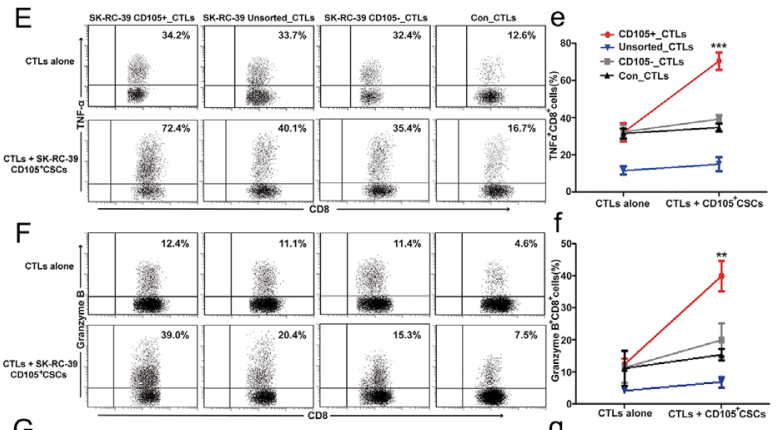

G
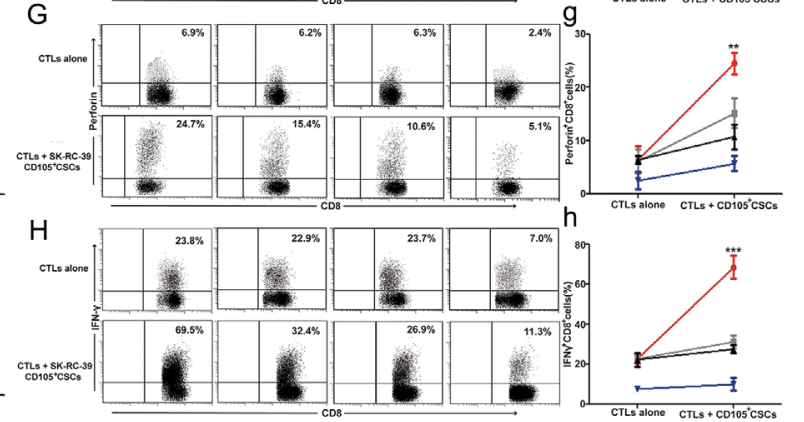

J

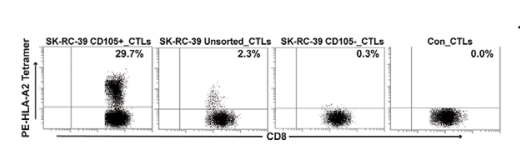

FIGURE 4 Dendritic cell loaded with CD105+ CSC lysate generate CSC-specific Th1 response. (A-D): TNF-a, Granzyme B, Perforin, and IFN- $\gamma$ release by $C D 8^{+}$CTLs after treatment with different $A 498$ cell lysate-loaded DCs. The portion of TNF- $a^{+} C D 8^{+}, G_{r a n z y m e ~} B^{+} C D 8^{+}$, Perforin ${ }^{+} \mathrm{CD}^{+}$, and IFN $-\gamma^{+} \mathrm{CD} 8^{+}$cells in A498 CD105+_CTLs significantly increased when cocultured with A498 CD105+ CSCs; however, the proportion of these Th1-related effector cells was not significantly changed in A498 Unsorted_CTLs, A498 CD105- _ CTLs, and Con_CTLs. (E-H): TNF- $\alpha$, Granzyme B, Perforin, and IFN- $\gamma$ release by CD8+ CTLs after treatment with different SK-RC-39 cell lysate-loaded DCs. The portion of TNF- $a+C D 8+$, Granzyme B + CD8+, Perforin + CD8+, and IFN- $\gamma+$ CD8+ cells in SK-RC-39 CD105 + CTLs significantly increased, when cocultured with SK-RC-39 CD105+ CSCs; however, the proportion of these Th1-related effector cells was not significantly changed in SK-RC-39 Unsorted_CTLs, SK-RC-39 CD105- _ CTLs, and Con_CTLs. Numbers represent frequencies (\%) of TNF- $a^{+}$, Granzyme $B^{+}$, Perforin $^{+}$, and IFN- $\gamma^{+}$cells in $C D 8^{+}$CTL populations. (a,b,c,d for A498 cell line and e,f,g,h for SK-RC-39 cell line): The mean value of increase in TNF- $a^{+}$, Granzyme $\mathrm{B}^{+}$, Perforin ${ }^{+}$, IFN- $\gamma^{+}$cells of $\mathrm{CD} 8^{+} \mathrm{CTLs}$ after coculture with CD105+ CSCs in the respective culture conditions in 3 independent experiments ${ }^{* *} P<0.01{ }^{* * *} P<0.001$. (I), A498 CD105+_CTLs could recognize specific CD105-associated antigen peptide; (J) SK-RC-39 CD105+_CTLs could recognize specific CD105-associated antigen peptide. Assessment of CD105-associated antigen-specific CTL clones by HLA/peptide tetramer staining. CD105+_CTLs, Unsorted_CTLs, CD105- _CTLs, and Con_CTLs clones $\left(1 \times 10^{5}\right.$ cells $)$ were stained with PEconjugated peptide/HLA tetramer at room temperature for $30 \mathrm{~min}$. Cells were then incubated with antibody against CD8 for $30 \mathrm{~min}$ at $4^{\circ} \mathrm{C}$. Cells were then examined by fluorescence-activated cell-sorting analysis using 10000 events/sample. Numbers represent frequencies (\%) of tetramer ${ }^{+}$cells in $\mathrm{CD}^{+}$CTL populations. The portion of tetramer + CD8+ cells in CD105+_CTLs was significantly higher than that in Unsorted_CTLs, CD105-_CTLs, and Con_CTLs. i,j, Mean value of tetramer+ cells of CD8+ CTL cells in the respective culture conditions in three independent experiments. ${ }^{* * *} P<0.001$

collected from A498 CD105- _DC, A498 Unsorted_DC, or Con_DC primed B cells. As shown in Fig. 5F, SK-RC-39 CD105+_DCs-primed immune supernatant bind to SK-RC-39 CD105+ CSCs significantly more than supernatant collected from SK-RC-39 CD105- _DC, SKRC-39 Unsorted_DC, or Con_DC primed B cells. To understand the immunological consequences of the binding of CD105+_DCs-primed antibody to CD105+ CSCs, we performed antibody and complementdependent cytotoxicity (CDC) assays directed against CSC targets. Immune supernatant collected from A498 CD105+_DCs-primed B cells were significantly more efficient mediators of A498 CD105+ CSC lysis (Fig. 6A). In contrast, immune supernatant collected from A498 Unsorted_DC-primed B cells and A498 CD105- _DC-primed B cells were significantly more efficient mediators of A498 Unsorted and A498 CD105- cell lysis, respectively (Fig. 6A). Immune supernatant collected from SK-RC-39 CD105+_DCs-primed B cells were significantly more efficient mediators of SK-RC-39 CD105+ CSC lysis (Fig. 6B). In contrast, immune supernatant collected from SK-RC-39 Unsorted_DC-primed B cells, and SK-RC-39 CD105- _DC-primed B cells were significantly more efficient mediators of SK-RC-39 Unsorted and SK-RC-39 CD105- cell lysis, respectively (Fig. 6B). 

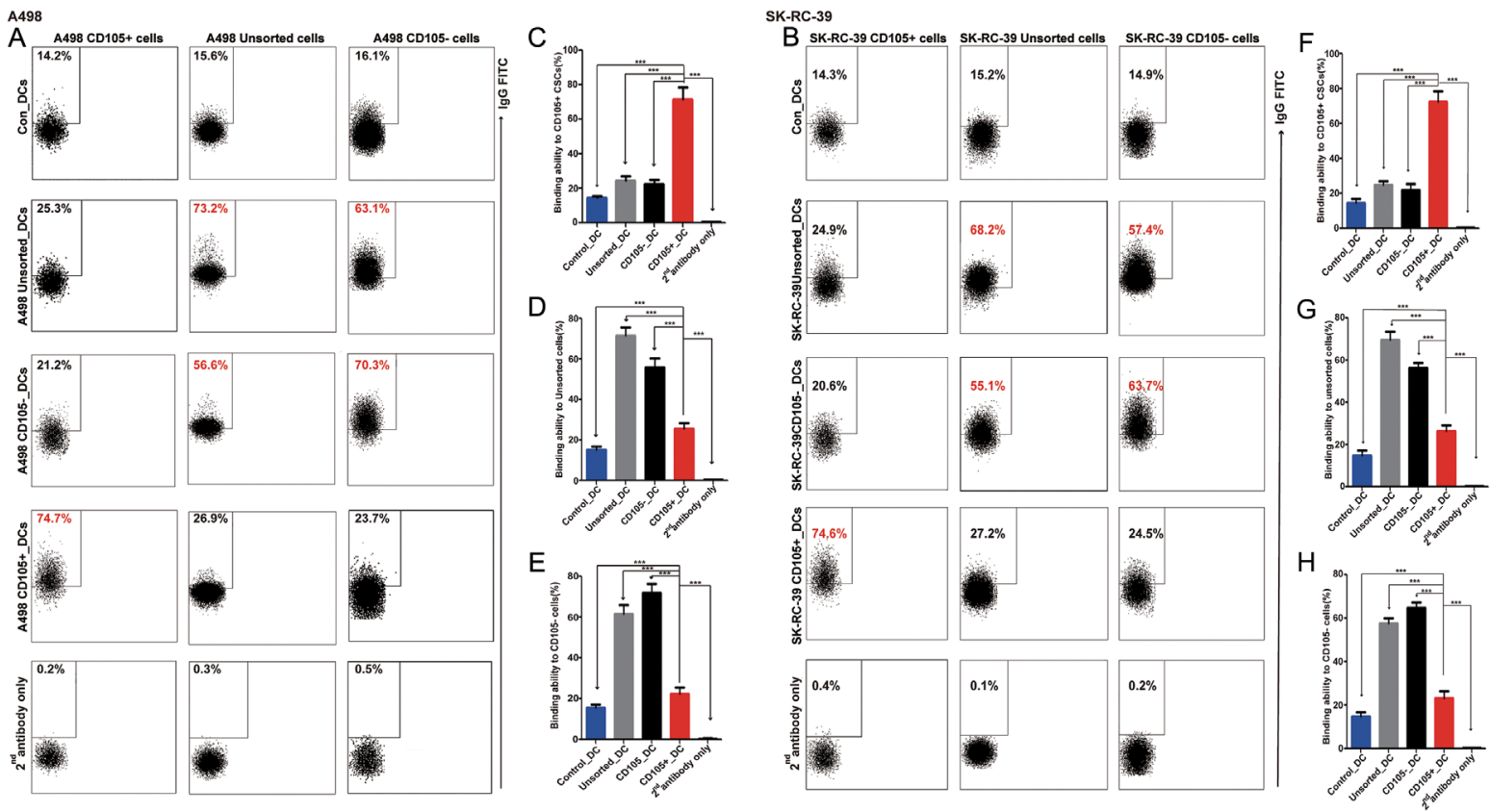

FIGURE 5 Co-culture of B cells with CD105+ DCs confers significant humoral response against renal cancer sem cells. (A) Specificity of the humoral immune response to CSCs was determined by assessing binding abilities to A498 CD105+ CSCs (left column), A498 Unsorted cells (middle column), A498 CD105- cells (right column) of the immune B cell culture supernatants collected from the culture of activated B cells cocultured with different A498 renal cancer cell lysate loaded DCs: Con_DCs, A498 Unsorted_DCs, A498 CD105-_DCs, and A498 CD105+_DCs. (B) Specificity of the humoral immune response to CSCs was determined by assessing binding abilities to SK-RC-39 CD105+ CSCs (left column), SK-RC-39 Unsorted cells (middle column), SK-RC-39 CD105- cells (right column) of the immune B cell culture supernatants collected from the culture of activated B cells cocultured with different SK-RC-39 renal cancer cell lysate loaded DCs: Con_DCs, SK-RC-39 Unsorted_DCs, SK-RC-39 CD105-_DCs, and SK-RC-39 CD105+_DCs. Secondary antibody only was used as negative control. (A, B): representative flow cytometry dot plot. (C) Statistical results from three repeated experiments showing different A498 renal cancer cell lysate loaded DCs activated B cells supernatants binding to A498 CD105+ CSCs, which was consistent with the left column of A. (D) Statistical results from three repeated experiments showing different A498 renal cancer cell lysate loaded DCs activated B cells supernatants binding to A498 Unsorted cells, which was consistent with the middle column of A; (E) Statistical results from three repeated experiments showing different A498 renal cancer cell lysate loaded DCs activated B cells supernatants binding to A498 CD105- cells, which was consistent with the right column of $A$; (F) Statistical results from three repeated experiments showing different SK-RC-39 renal cancer cell lysate loaded DCs activated B cells supernatants binding to SK-RC-39 CD105+ CSCs, which was consistent with the left column of B; (G) Statistical results from three repeated experiments showing different SK-RC-39 renal cancer cell lysate loaded DCs activated B cells supernatants binding to SK-RC-39 Unsorted cells, which was consistent with the middle column of B; $(\mathrm{H})$ Statistical results from three repeated experiments showing different SK-RC-39 renal cancer cell lysate loaded DCs activated B cells supernatants binding to SK-RC-39 CD105- cells; which was consistent with the right column of $B$

These data support the conclusion that CD105+_DCs confer significant host anti-CSC immunity by producing CD105+ CSCspecific antibodies, which bind and kill CD105+ CSCs.

\section{4 | DISCUSSION}

Current therapeutic strategies against cancer have serious limitations that frequently lead to treatment failure. A common cause of treatment failure in multiple malignancies is resistance to chemotherapy, radiotherapy, and immunotherapy. In addition, many strategies that can be toxic to differentiated cancer cells are not sufficiently selective against CSCs; patients therefore face the risk of recurrence and metastasis because of CSC persistence. ${ }^{48}$ CSC populations are more resistant to conventional cancer therapies than non-CSC populations are. Therefore, the elimination of CSCs is crucial in treating malignant diseases. ${ }^{49-57}$ Thus, studies on CSC-targeted therapeutic regimens, which could lead to complete eradication of cancer, are of great significance.

In recent years, there has been a focus on the identification and characterization of CSCs in most malignant tumors including human RCC. Bussolati et al ${ }^{11}$ firstly demonstrated that CD105+ RCC primary cancer cells possessed tumorigenicity and self-renewal through detecting their in vivo transplantation and serial propagations at limited dilutions. Similar to the results of Bussolati et al, we found that sorted CD105+ cell subpopulations from A498 and SK-RC-39 RCC cell lines were able to form non-adherent spheroids in SFM in the presence of EGF and bFGF, and could produce a tumor mass with few cell number when transplanted into immunodeficient mice. Thus, CD105+ subpopulation was considered to be RCC CSCs in our further investigation. 


\section{A $\quad \mathbf{A 4 9 8}$}

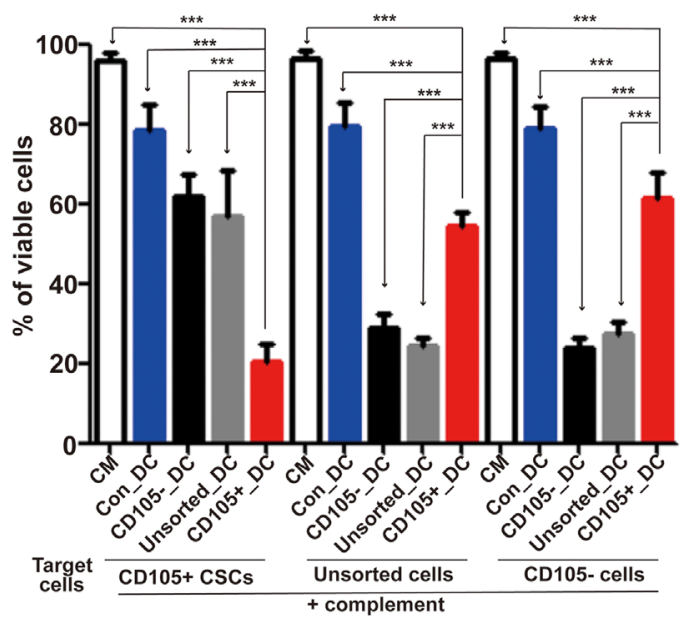

B SK-RC-39

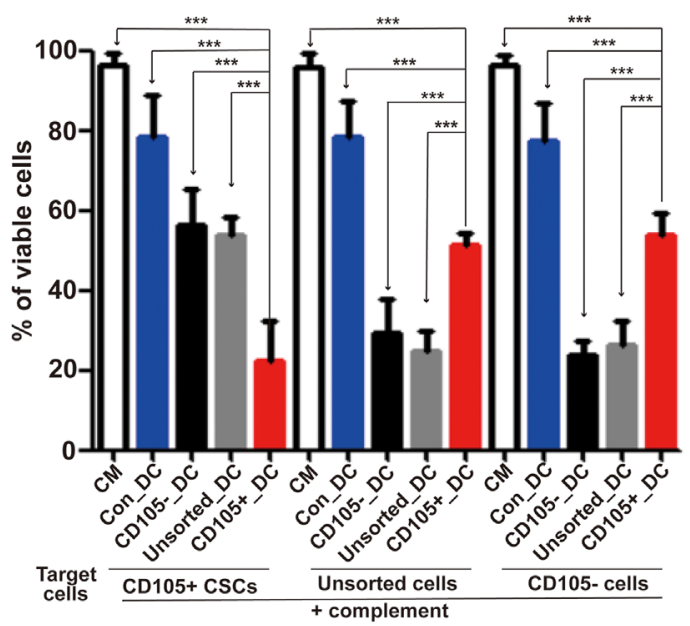

FIGURE 6 Antibody generated by B cells cocultured with CD105 + DCs could target CSCs selectively via complement-dependent cytotoxicity (CDC). (A) Antibody and complement-mediated cytotoxicity was measured by incubating A498 CD105+ cancer stem cells (CSCs), A498 unsorted cancer cells, or A498 CD105non-CSCs with immune B-cell culture supernatant collected from the culture of activated B cells co-cultured with A498 CD105 +_DCs, A498 Unsorted_DCs, A498 CD105- _DCs, Con-DCs, respectively. (B) Antibody and complement-mediated cytotoxicity was measured by incubating SK-RC-39 CD105+ cancer stem cells (CSCS), SK-RC-39 unsorted cancer cells, or SK-RC-39 CD105- nonCSCs with immune B-cell culture supernatant collected from the culture of activated B cells co-cultured with SK-RC-39 CD105 + _DCs, SK-RC-39 Unsorted_DCs, SK-RC-39 CD105- _DCs, ConDCs, respectively. Data are expressed as the percentages of viable cells. The lower the percentage of viable cells, the higher the percentage of cell lysis. Data are representative of three experiments. ${ }^{* * *} P<0.001$

Recently, cancer immunotherapy strategies attempting to direct the immune system to eradicate tumor initiating and maintaining CSCs have been developed. ${ }^{52}$ Tumor recurrence could be prevented if CSCs are specifically killed. CSC-targeting immunotherapy is potentially the most effective strategy to treat a wide range of cancers. Several reports have shown that CSCs can be eradicated by innate immune effector cells, such as natural killer (NK) cells and $\gamma \delta T$ lymphocytes. Castriconi et al ${ }^{55}$ demonstrated that stem-like cells are highly susceptible to lysis by allogeneic and autologous activated NK cells. Besides, DCs, the most powerful antigen presenting cells, have been used to induce effective antitumor immune responses in experimental animals and numerous clinical trials. ${ }^{58-61}$ Fields et al, ${ }^{60}$ reported that bone marrow-derived DCs loaded with tumor lysates could induce CTL and T cell proliferative responses. Moreover, several other studies also suggested that vaccination with autologous DCs arising from peripheral blood monocytes is a safe and promising approach for the treatment of renal cancer. ${ }^{29-34}$ Our results demonstrated that human PBMC-derived DCs are powerful immunizers, and can mediate anti-tumor immune responses against RCC CSCs. Furthermore, we demonstrated that the loading of DCs with RCC CSC lysates as tumor antigen more efficiently directs CTL responses toward CSCs. These results were similar to Pellegatta et al, ${ }^{30}$ who showed that splenocytes from mice immunized with malignant glioma CSC-loaded DCs had specific lytic activity against glioma CSC. Additionally, we reported previously that the DC-based vaccination could be used to selectively target CSCs and confer antitumor immunity. ${ }^{24}$ These proof-ofconcept results provide a rationale for a novel cancer immunotherapy method based on the development of CSC-DC vaccines that can specifically target CSCs. ${ }^{25}$ Consistent with the above reports, our present results showed that CSC_CTLs directly target CSCs. The CTL reaction is a potent response, inducing potential protective immunity against nearly all tumors. ${ }^{53,54}$ The present study demonstrated that CD105+ CSC-loaded DCs induced greater numbers of functionally active T cells. Further, these effector T cells preferentially killed CD105+ RCC cells, accompanied by a decrease in the frequency of the CD105+ subpopulation within the RCC bulk population both in vitro and in vivo. Our results showed that CSC lysate-loaded DCs not only induce more efficient CTL activity against CSCs, but also increase the IFN- $\gamma$ secretion, indicating a shift to the $\mathrm{TH} 1$ phenotype. A switch from TH1 to $\mathrm{TH} 2$ profiles could result in progressive tumor growth in a wide variety of murine malignancies, including $B$ cell leukemia/lymphoma, melanoma, RCC, and colon adenocarcinoma. Xu et al $^{62}$ also showed that CSC-pulsed DCs induce the antigen-specific TH1 immune response. However, they found that mice immunized against glioma cells depleted of CSCs produce significantly less INF- $\gamma$ than those immunized with CSC-pulsed DCs. Consistent with their results, we observed that, vaccination with DCs loaded with RCC CSCs, but not the non-CSC cells or unsorted cells, induced CTLs that recognized CSCs. In addition, when co-cultured with CD105+ CSCs, the portion of IFN- $\gamma+$ CD8+ cells were significantly higher in CD105+_CTLs than in Unsorted_CTLs, CD105-_CTLs, and Con_CTLs. Furthermore, we found that CD105+ CSC-loaded DCs could induce humoral anti-CSC immunity in vitro. We observed specific binding of CD105+ CSCs by lgG produced by CD105+_DCs-primed B cells. Importantly, specific binding of IgG produced by CD105+_DCs-primed B cells to CD105+ CSCs resulted in significant lysis of the target cells in the presence of complement. This is similar with our previous reports, ${ }^{24,25}$ which showed that CSC lysatepulsed DCs could significantly induce more CSCs specific antibody, compared to whole D5 tumor cell lysate-pulsed DCs. 


\section{5 | CONCLUSION}

The CSC hypothesis is well established and widely accepted, ${ }^{63}$ and postulates that targeted ablation of CSCs could circumvent the problem of therapeutic resistant cells and consequent tumor relapse. Our results showed that CSC-targeted DC therapy could be a potent strategy for CSC elimination and therefore prevention of tumor metastasis and relapse after conventional tumor therapies. However, much of the research on CSC-targeted DC based therapy is preliminary, and more studies are required to clarify this attractive field.

\section{ACKNOWLEDGMENTS}

This work was primarily supported by grants from the National Natural Science Foundation of China (Nos. 81472387, 81402560), Guangdong Science and Technology Program (No. 2013B021800063), and was partially supported by the Guangdong Province Science and Technology Plan Project (No. 2012A030400059), and by the Gillson Longenbaugh Foundation.

\section{DISCLOSURES}

The authors report no conflict of interest.

\section{ORCID}

Jian-Chuan Xia iD http://orcid.org/0000-0002-1268-3967

\section{REFERENCES}

1. Theodoropoulos PA, Polioudaki H, Agelaki S, et al. Circulating tumor cells with a putative stem cell phenotype in peripheral blood of patients with breast cancer. Cancer Lett. 2010;288:99-106.

2. Clevers $\mathrm{H}$. The cancer stem cell: premises, promises and challenges. Nat Med. 2011;17:313-319.

3. Bjerkvig R, Johansson M, Miletic H, Niclou SP. Cancer stem cells and angiogenesis. Semin Cancer Biol. 2009;19:279-284.

4. Kreso A, Dick JE. Evolution of the cancer stem cell model. Cell Stem Cell. 2014;14:275-291.

5. Al-Hajj M, Wicha MS, Benito-Hernandez A, et al. Prospective identification of tumorigenic breast cancer cells. Proc Natl Acad Sci USA. 2003;100:3983-3988.

6. Singh SK, Hawkins C, Clarke ID, et al. Identification of human brain tumour initiating cells. Nature. 2004;432:396-401.

7. Li C, Heidt DG, Dalerba P, et al. Identification of pancreatic cancer stem cells. Cancer Res. 2007;67:1030-1037.

8. Collins AT, Berry PA, Hyde C, et al. Prospective identification of tumorigenic prostate cancer stem cells. Cancer Res. 2005;65: 10946-10951.

9. Bapat SA, Mali AM, Koppikar CB, et al. Stem and progenitor-like cells contribute to the aggressive behaviour of human epithelial ovarian cancer. Cancer Res. 2005;65:3025-3029.

10. Fang D, Nguyen TK, Leishear K, et al. A tumorigenic subpopulation with stem cell properties in melanomas. Cancer Res. 2005;65: 9328-9337.
11. Bussolati B, Bruno S, Grange C, et al. Identification of a tumor-initiating stem cell population in human renal carcinomas. FASEB J. 2008;22: 3696-3705.

12. Myszczyszyn A, Czarnecka AM, Matak D, et al. The role of hypoxia and cancer stem cells in renal cell carcinoma pathogenesis. Stem Cell Rev Rep. 2015;11:919-943.

13. Axelson $\mathrm{H}$, Johansson ME. Renal stem cells and their implications for kidney cancer. Semin Cancer Biol. 2013;23:56-61.

14. Bu Y, Cao D. The origin of cancer stem cells. Fronti Biosci. 2012;4: 819-830.

15. Shen H, Boyer M, Cheng T. Flow cytometry-based cell cycle measurement of mouse hematopoietic stem and progenitor cells. Methods Mol Biol. 2008;430:77-86.

16. Klonisch T, Wiechec E, Hombach-Klonisch S, et al. Cancer stem cell markers in common cancers-therapeutic implications. Trends $\mathrm{Mol}$ Med. 2008;14:450-460.

17. Wang Z, Li Y, Ahmad A, et al. Targeting miRNAs involved in cancer stem cell and EMT regulation: an emerging concept in overcoming drug resistance. Drug Resist Updat. 2010;13:109-118.

18. Liu J, Kopecková $P$, Bühler $P$, et al. Biorecognition and subcellular trafficking of HPMA copolymer-anti-PSMA antibody conjugates by prostate cancer cells. Mol Pharm. 2009;6:959-970.

19. Curiel TJ. Immunotherapy: a useful strategy to help combat multidrug resistance. Drug Resist Updates. 2012;15:106-113.

20. Jin L, Hope KJ, Zhai Q, et al. Targeting of CD44 eradicates human acute myeloid leukemic stem cells. Nat Med. 2006;12:1167-1174.

21. Jin L, Lee EM, Ramshaw HS, et al. Monoclonal antibody-mediated targeting of CD123, IL-3 receptor a chain, eliminates human acute myeloid leukemic stem cells. Cell Stem Cell. 2009;5:31-42.

22. Kikushige $Y$, Shima T, Takayanagi S, et al. TIM-3 is a promising target to selectively kill acute myeloid leukemia stem cells. Cell Stem Cell. 2010;7:708-717.

23. Chao MP, Alizadeh AA, Tang C, et al. Therapeutic antibody targeting of CD47 eliminates human acute lymphoblastic leukemia. Cancer Res. 2011;71:1374-1384.

24. Ning N, Pan Q, Zheng F, et al. Cancer stem cell vaccination confers significant antitumor immunity. Cancer Res. 2012:72:1853-1864.

25. Lu L, Tao H, Chang AE, et al. Cancer stem cell vaccine inhibits metastases of primary tumors and induces humoral immune responses against cancer stem cells. Oncoimmunology. 2015;4:e990767.

26. Boiko AD, Razorenova OV, van de Rijn M, et al. Human melanomainitiating cells express neural crest nerve growth factor receptor CD271. Nature. 2010:466:133-137.

27. Busse A, Letsch A, Fusi $A$, et al. Characterization of small spheres derived from various solid tumor cell lines: are they suitable targets for T cells. Clin Exp Metastasis. 2013;30:781-791.

28. Kantoff PW, Higano CS, Shore ND, et al. Sipuleucel-T immunotherapy for castration-resistant prostate cancer. New Eng J Med. 2010;363: 411-422.

29. Su Z, Dannull J, Heiser A, et al. Immunological and clinical responses in metastatic renal cancer patients vaccinated with tumor RNA-transfected dendritic cells. Cancer Res. 2003;63:2127-2133.

30. Pellegatta S, Poliani PL, Corno D, et al. Neurospheres enriched in cancer stem-like cells are highly effective in eliciting a dendritic cellmediated immune response against malignant gliomas. See comment in PubMed Commons below. Cancer Res. 2006;66:10247-10252.

31. Kim JH, Lee Y, Bae YS, et al. Phase I/II study of immunotherapy using autologous tumor lysate-pulsed dendritic cells in patients with metastatic renal cell carcinoma. Clin Immunol. 2007;125:257-267.

32. Berntsen A, Trepiakas R, Wenandy L, et al. Therapeutic dendritic cell vaccination of patients with metastatic renal cell carcinoma: a clinical phase 1/2 trial. J Immunother. 2008;31:771-780.

33. Schwaab T, Schwarzer A, Wolf B, et al. Clinical and immunologic effects of intranodal autologous tumor lysate-dendritic cell vaccine 
with aldesleukin (interleukin 2) and IFN-a2a therapy in metastatic renal cell carcinoma patients. Clin Cancer Res. 2009;15:4986-4992.

34. Flörcken A, Kopp J, van Lessen A, et al. Allogeneic partially HLAmatched dendritic cells (DC) as a vaccine in metastatic renal cell cancer (mRCC): final analysis of a clinical phase I/II study. J Clin Oncol. 2012;30:e15053.

35. Hirohashi Y, Torigoe T, Inoda S, et al. Immune response against tumor antigens expressed on human cancer stem-like cells/tumor-initiating cells. Immunotherapy. 2010;2:201-211.

36. Li X, Zhang Z, Lin G, et al. Antigen-specific T-cell response from dendritic cell vaccination using cancer stem-like cell-associated antigens. Stem Cells. 2009;27:1734-1740.

37. Weng D, Song B, Durfee J, et al. Induction of cytotoxic T lymphocytes against ovarian cancer-initiating cells. Int J Cancer. 2011;129:1990-2001.

38. Sun JC, Pan K, Chen MS, et al. Dendritic cells-mediated CTLs targeting hepatocellular carcinoma stem cells. Cancer Biol Ther. 2010;10: 368-375.

39. Pan QZ, Pan K, Wang QJ, et al. Annexin A3 as a potential target for immunotherapy of liver cancer stem-like cells. Stem Cells. 2015;33:354-366.

40. Dallas NA, Samuel S, Xia L, et al. Endoglin (CD105): a marker of tumor vasculature and potential target for therapy. Clin Cancer Res. 2008;14:1931-1937.

41. Hu D, Wang X, Mao Y, et al. Identification of CD105 (endoglin)positive stem-like cells in rhabdoid meningioma. J Neurooncol. 2012;106:505-517.

42. Ziebarth AJ, Nowsheen S, Steg AD, et al. Endoglin (CD105) contributes to platinum resistance and is a target for tumor-specific therapy in epithelial ovarian cancer. Clin Cancer Res. 2013;19:170-182.

43. Tomuleasa C, Soritau O, Rus-Ciuca D, et al. Functional and molecular characterization of glioblastoma multiforme-derived cancer stem cells. J BUON. 2010;15:583-591.

44. Royer-Pokora B, Busch M, Beier M, et al. Wilms tumor cells with WT1 mutations have characteristic features of mesenchymal stem cells and express molecular markers of paraxial mesoderm. Hum Mol Genet. 2010;19:1651-1668.

45. Huang AH, Chen YK, Chan AW, et al. Isolation and characterization of normal hamster buccal pouch stem/stromal cells-potential oral cancer stem/stem-like cell model. Oral Oncol. 2009;45:e189-e195.

46. Chen W, Dong J, Haiech J, et al. Cancer stem cell quiescence and plasticity as major challenges in cancer therapy. Stem Cells Int. 2016;2016:1740936.

47. Crea F, Danesi R, Farrar WL. Cancer stem cell epigenetics and chemoresistance. Epigenomics. 2009;1:63-79.

48. Zhao J. Cancer stem cells and chemoresistance: the smartest survives the raid. Pharmacol Ther. 2016;160:145-158.

49. Norazizah Shafee, Christopher Smith R, Shuanzeng Wei, et al. Cancer stem cells contribute to cisplatin resistance in Brca1/p53-Mediated mouse mammary tumors. Cancer Res. 2008;68:3243-3250.

50. Cho K, Wang X, Nie S, et al. Therapeutic nanoparticles for drug delivery in cancer. Clin Cancer Res. 2008;14:1310-1316.

51. LaBarge MA. The difficulty of targeting cancer stem cell niches. Clin Cancer Res. 2010;16:3121-3129.
52. Lacerda L, Pusztai L, Woodward WA. The role of tumor initiating cells in drug resistance of breast cancer: implications for future therapeutic approaches. Drug Resist Updat. 2010;13:99-108.

53. Sharma RK, Chheda ZS, Jala VR, et al. Regulation of cytotoxic TLymphocyte trafficking to tumors by chemoattractants: implications for immunotherapy. Expert Rev Vaccines. 2015;14:537-549.

54. Lesterhuis WJ, Haanen JB, Punt CJ. Cancer immunotherapy-revisited. Nat Rev Drug Discov. 2011;10:591-600.

55. Wang Z, Li Y, Ahmad A, et al. Targeting miRNAs involved in cancer stem cell and EMT regulation: an emerging concept in overcoming drug resistance. Drug Resist Updat. 2010;13:109-118.

56. Zhong Y, Guan K, Guo S, et al. Spheres derived from the human SK-RC42 renal cell carcinoma cell line are enriched in cancer stem cells. Cancer Lett. 2010;299:150-160.

57. Castriconi R, Daga A, Dondero A, et al. NK cells recognize and kill human glioblastoma cells with stem cell-like properties. J Immunol. 2009;182:3530-3539.

58. de Vries IJ, Lesterhuis WJ, Barentsz JO, et al. Magnetic resonance tracking of dendritic cells in melanoma patients for monitoring of cellular therapy. Nar Biotech. 2005;23:1407-1413.

59. Nestle FO, Alijagic S, Gilliet M, et al. Vaccination of melanoma patients with peptide- or tumorlysate-pulsed dendritic cells. Nat Med. 1997;4: 328-333.

60. Fields RC, Shimizu K, Mulé JJ. Murine dendritic cells pulsed with whole tumor lysates mediate potent antitumor immune responses in vitro and in vivo. Proc Natl Acad Sci USA. 1998;95:9482-9487.

61. Banchereau J, Palucka AK, Dhodapkar M, et al. Melanoma to CD34 + progenitor-derived dendritic cell vaccine immune and clinical responses in patients with metastatic. Cancer Res. 2001;61:6451-6458.

62. Xu Q, Liu G, Yuan X, et al. Antigen-specific-cell response from dendritic cell vaccination using cancer stem-like cellassociated antigens. Stem Cells. 2009;27:1734-1740.

63. Adorno-Cruz V, Kibria G, Liu X, et al. Cancer stem cells: targeting the roots of cancer, seeds of metastasis, and sources of therapy resistance. Cancer Res. 2015;75:924-929.

\section{SUPPORTING INFORMATION}

Additional Supporting Information may be found online in the supporting information tab for this article.

How to cite this article: Zhang X-F, Weng D-s, Pan K, et al. Dendritic-cell-based immunotherapy evokes potent antitumor immune responses in CD105+ human renal cancer stem cells. Molecular Carcinogenesis. 2017;56:2499-2511. https://doi.org/10.1002/mc.22697 